\title{
Perfil das Intoxicações Medicamentosas Notificadas ao SINAN no Município de Primavera do Leste - MT, Entre os Anos de 2007 a 2014
}

\section{Profile of Medicinal Intoxications Notified at SINAN in the Municipality of Primavera do Leste - MT, Between the Years 2007 to 2014}

\author{
Vivian Tallita Pinheiro de Santana ${ }^{\mathrm{a}}$; Eliane Aparecida Suchara ${ }^{\mathrm{b}}$; Roberta Carreto*c; Phelipe Magalhães Duarte ${ }^{\mathrm{a}}$
}

aUniversidade de Cuiabá. MT, Brasil.

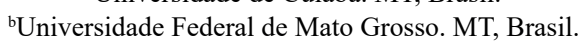

'Instituto Universitário Italiano de Rosário, Programa de Pós-Graduação Stricto Sensu em Ciências Biomédicas, Argentina.

*E-mail: roberta.carreto@kroton.com.br

\begin{abstract}
Resumo
O conhecimento do perfil epidemiológico das intoxicações medicamentosas é um importante guia para a gestão de recursos destinados ao planejamento e à implementação de ações que previnam a ocorrência desse agravo. O presente trabalho buscou estudar o perfil das intoxicações medicamentosas ocorridas no município de Primavera do Leste-MT, entre 2007 a 2014. Foram avaliados dados secundários registrados no Sistema de Informação de Agravos de Notificação (SINAN), que permitiram a realização de um estudo epidemiológico descritivo e transversal em que se analisaram informações referentes ao perfil das vítimas (idade, sexo) e dos casos (circunstâncias, tipo de exposição e evolução dos casos). Registraram-se 52 casos, destes $73,1 \%(n=38)$ são vítimas do gênero feminino e $26,9 \%$ ( $n=14)$ do masculino. As intoxicações com medicamentos ocorreram em quase todas as faixas etárias ( 01 a 64 anos), porém a maioria das vítimas possuía entre 20 a 39 anos (n=26; $50 \%)$ e 15 a $19(17,3 \% ; n=9)$. As circunstâncias relacionadas às intoxicações foram: $73,1 \%(n=38)$ por tentativa de suicídio, $15,4 \%(n=8)$ por uso acidental e os demais pelo uso habitual, abuso, automedicação e violência/homicídio. Em 82,7\% ( $\mathrm{n}=43$ ) dos casos, as exposições foram agudas e únicas, em 9,6\% (n=5) agudas repetidas, não sendo registrada exposição crônica. 84,6\% $(\mathrm{n}=44)$ das intoxicações evoluíram para cura sem sequelas. Registrou-se um óbito em função da tentativa de suicídio. As intoxicações por medicamentos são frequentes e representam um importante problema de saúde pública, que necessita de ações preventivas e educativas com foco nas vítimas e circunstâncias mais frequentes. Palavras-chave: Suicídio. Abuso de Medicamentos. Automedicação.
\end{abstract}

\begin{abstract}
Knowledge of the epidemiological profile of drug intoxications (Notification of Injury Information System) is an important guide for the resources management intended to the planning and implementation of actions that prevent the occurrence of this outcome. The present study aimed to describe the drug intoxications profile occurring in the municipality of Primavera do Leste-MT, between 2007 and 2014. Secondary data recorded in SINAN (Information System for Notifiable Diseases) were used to conduct a descriptive and cross - sectional epidemiological study in which information was analyzed regarding the victims 'profile (age, sex) and cases (circumstances, type of exposure and evolution of cases). There were 52 cases, of which $73.1 \%(n=38)$ were females and $26.9 \%(n=14)$ males. Drug intoxications occurred in almost all age groups (01 to 64 years), but the most of the were between 20 and 39 years $(n=26 ; 50 \%)$ and 15 to $19(17.3 \%$; $n=9)$. The circumstances related to intoxication were: $73.1 \%(n=38)$ for attempted suicide, $15.4 \%(n=8)$ for accidental use and the others for habitual use, abuse, self-medication and violence / homicide. In $82.7 \%(n=43)$ of the cases the exposures were acute and single, in 9.6\% $(n=5)$ repeated acute, and chronic exposure was not recorded. $84.6 \%(n=44)$ of intoxications evolved to cure without sequelae. A death was recorded due to suicide attempt. Drugs intoxications are frequent and represent a relevant public health problem that requires preventive and educational actions with a focus on the most frequent victims and circumstances.
\end{abstract}

Keywords: Suicide. Drug Misuse.Self-Medication.

\section{Introdução}

A saúde dos organismos vivos pode sofrer danos em casos de exposição desses a algumas substâncias comuns ao dia a dia, tais como: produtos químicos de higiene e limpeza, medicamentos, cosméticos, defensivos agrícolas, toxinas, peçonhas, plantas tóxicas, entre outras (MOREIRA et al., 2010). Assim, o contato do organismo vivo com estas substâncias químicas pode resultar em intoxicação com prejuízo para a saúde e risco à vida (SCHVARTSMAN; SCHVARTSMAN, 1999).

As intoxicações são observadas pela manifestação dos efeitos nocivos produzidos quando uma substância tóxica entra em contato com o organismo através de ingestão, aspiração, contato com a pele, olhos ou mucosas e, caracterizam-se pelo aparecimento dos efeitos tóxicos, tendo como consequência um processo patológico caracterizado por desequilíbrio fisiológico decorrente de alterações bioquímicas no organismo, o que pode ser evidenciado por sinais e sintomas ou mediante exames laboratoriais (OGA et al., 2008; SCHVARTSMAN; SCHVARTSMAN, 1999).

Entre os agentes químicos existentes, os medicamentos representam uma das principais causas de intoxicações humanas registradas no Brasil, e o crescimento do registro desses casos vem sendo destacado pelo Sistema Nacional 
de Informações Tóxico-Farmacológicas (SINITOX) em publicações de relatórios de notificações anuais (SINITOX; BRASIL, 2007; FIOCRUZ, 2010a).

A ocorrência de intoxicações medicamentosas, possivelmente, está relacionada com o padrão de consumo de medicamentos do Brasil, que é fortemente influenciado por falhas no controle do uso, inadequações na produção e comercialização de produtos farmacêuticos, levando ao consumo abusivo e irracional de produtos de venda livre e sob prescrição, resultando no crescimento de casos de intoxicação e envenenamentos (ROZENFELD, 1998; MATOS et. al., 2008).

Entre as razões para maior prevalência de intoxicação por medicamentos, também se destacam a fácil disponibilidade em domicílios, o amplo uso sem orientação médica e o uso indiscriminado de medicamentos e associações de fármacos, bem como a extensa variedade de medicamentos existentes no Brasil (MERT; BILGIN, 2006; ARRAIS, 2002; WONG, 2003).

Tais situações favorecem o surgimento de problemas relacionados ao uso destes produtos e contribuem para que os medicamentos constituam o principal agente tóxico responsável por intoxicações humanas registrados no país (LESSA; BOCHNER, 2008; BRASIL, 2011b). As intoxicações medicamentosas constituem, assim, um grave problema de saúde pública (BERTASSO-BORGES et al., 2010).

No Brasil, os casos de intoxicação registrados são disponibilizados através de publicações anuais do SINITOX. Esse sistema agrupa as informações registradas em 36 Centros de Controle de Intoxicações (CCI), localizados em 19 estados e no DF (PRESGRAVE et al., 2009).

Todos os casos de intoxicação ocorridos devem ser notificados e registrados através do Sistema de Informação de Agravos de Notificação (SINAN). A obrigatoriedade da notificação é estabelecida para toda rede de saúde, tanto pública quanto privada (Portaria $\mathrm{n}^{\circ} 114$ de 25 de janeiro de 2011 do Ministério da Saúde) e deve ser aplicada a todas as doenças e eventos constantes na Lista de Notificação Compulsória (LNC) (BRASIL, 2011a). No entanto, apesar do registro ser compulsório, nem todos os casos ocorridos são verdadeiramente notificados, principalmente, em função da tendência de registrar apenas os mais agudos, com sinais clínicos mais graves (MATOS et al., 2002).

O conhecimento do perfil epidemiológico das intoxicações medicamentosas nos municípios e demais unidades federativas é um importante guia para a gestão de recursos destinados ao planejamento e à implementação de ações que previnam a ocorrência desse agravo (SILVA, 2009). As informações epidemiológicas fornecidas por esses dados registrados são de grande interesse social, pois oferecem elementos úteis para a realização de pesquisas e correspondem a importantes fontes de informações, que podem ser empregadas em estudos epidemiológicos e na vigilância de doenças e agravos à saúde (COELI; CAMARGO JR., 2002).

Visando contribuir para a adoção de medidas preventivas e para o planejamento em saúde, o presente estudo buscou caracterizar as intoxicações medicamentosas notificadas ao SINAN, com o objetivo de identificar o perfil das vítimas, as principais circunstâncias, os tipos de exposição e a evolução dos casos de intoxicações medicamentosas registradas no município de Primavera do Leste - MT.

\section{Material e Métodos}

Trata-se de um estudo epidemiológico descritivo e transversal dos casos de intoxicações medicamentosas registrados entre 2007 a 2014 no município de Primavera do Leste - MT, junto ao SINAN. Esse estudo compõe projeto de pesquisa aprovado pelo Comitê de Ética da Universidade Federal de Mato Grosso sob o número do CAAE (Certificado de Apresentação para Apreciação Ética) 24213113.3.0000.5587.

Para o estudo foram inclusas todas as notificações registradas na base do SINAN, entre os anos de 2007 a 2014. Foram avaliados os seguintes parâmetros: sexo, idade e raça/ cor das vítimas; as principais circunstâncias das intoxicações: acidental, intencional (tentativa de suicídio), automedicação, uso abusivo, uso habitual violência/homicídio, sem identificação de circunstância; os tipos de exposição, aguda ou crônica; a evolução dos casos de intoxicações medicamentosas para cura e ou óbitos. Os dados foram tabulados por meio do programa Excel para gerar as tabelas, as figuras e realizar cálculos de frequências absolutas $(\mathrm{N})$ e relativas (\%) para posterior análise.

\section{Resultados e Discussão}

Ao longo dos anos avaliados foram registrados 52 casos de intoxicações por medicamentos ocorridas no município de Primavera de Leste - MT. Maior número de notificações foi encontrado em 2008 (25\%; n=13) e 2012 (19,2\%; $\mathrm{n}=10$ ). É possível que outros casos não notificados tenham ocorrido durante o período avaliado, porém mesmo com a obrigatoriedade dos registros, a subnotificação ainda constitui um problema em todo o país (MATOS et al., 2002).

As vítimas de intoxicação no município foram em maior proporção do gênero feminino $(26,9 \%$; $=38)$ (Quadro 1). No ano de 2010, o SINITOX relatou que vítimas femininas estiveram envolvidas em maior número de casos ocorridos na Região Centro-Oeste (FIOCRUZ, 2010b). No estudo de Bortoletto e Bochner (1999), a participação do sexo feminino também foi bastante expressiva, tendo sido observado que mais da metade (63\%) das vítimas de intoxicação por medicamentos correspondeu a este gênero. 
Quadro 1 - Registros de intoxicação medicamentosa no município de Primavera do Leste - MT, 2007 a 2014.

\begin{tabular}{|c|c|c|}
\hline Ano de Ocorrência & Número de Casos & $\%$ \\
\hline 2007 & 0 & 0,0 \\
\hline 2008 & 13 & 25,0 \\
\hline 2009 & 6 & 11,5 \\
\hline 2010 & 9 & 17,3 \\
\hline 2011 & 2 & 3,8 \\
\hline 2012 & 10 & 19,2 \\
\hline 2013 & 7 & 13,5 \\
\hline 2014 & 5 & 9,6 \\
\hline Total & 52 & 100,0 \\
\hline \multicolumn{3}{|l|}{ Gênero } \\
\hline Masculino & 14 & 26,9 \\
\hline Feminino & 38 & 73,1 \\
\hline Total & 52 & 100,0 \\
\hline \multicolumn{3}{|l|}{ Faixas etárias } \\
\hline$<1$ Ano & & 0,0 \\
\hline 01 à 04 & 5 & 9,6 \\
\hline 05 à 09 & 1 & 1,9 \\
\hline 10 à 14 & 5 & 9,6 \\
\hline 15 à 19 & 9 & 17,3 \\
\hline 20 à 39 & 26 & 50,0 \\
\hline 40 à 59 & 5 & 9,6 \\
\hline 60 à 64 & 1 & 1,9 \\
\hline Total & 52 & 100,0 \\
\hline \multicolumn{3}{|l|}{ Circunstâncias } \\
\hline Acidental & 8 & 15,4 \\
\hline Tentativa de suicídio & 38 & 73,1 \\
\hline Uso Habitual & 1 & 1,9 \\
\hline Ignorado/Branco & 2 & 3,8 \\
\hline Abuso & 1 & 1,9 \\
\hline Automedicação & 1 & 1,9 \\
\hline Violência/homicídio & 1 & 1,9 \\
\hline Total & 52 & 100,0 \\
\hline \multicolumn{3}{|l|}{ Tipo de exposição } \\
\hline Aguda-única & 43 & 82,7 \\
\hline Aguda-repetida & 5 & 9,6 \\
\hline Crônica & & 0,0 \\
\hline Ign/Branco & 4 & 7,7 \\
\hline Total & 52 & 100,0 \\
\hline \multicolumn{3}{|l|}{ Evolução } \\
\hline Ign/Branco & 7 & 13,5 \\
\hline Cura sem sequela & 44 & 84,6 \\
\hline Cura com sequela & 0 & 0,0 \\
\hline $\begin{array}{l}\text { Óbito por intoxicação } \\
\text { Exógena }\end{array}$ & 1 & 1,9 \\
\hline Total & 52 & 100,0 \\
\hline
\end{tabular}

Fonte: Dados da pesquisa.

A maior ocorrência de intoxicações entre indivíduos do gênero feminino pode estar relacionada ao comportamento ocupacional da mulher moderna que, em geral, apresenta dupla jornada que associa o trabalho externo com as atividades domésticas. Essa condição pode induzir a automedicação um uso acentuado de medicamentos em função da necessidade de estar sempre bem-disposta para cumprir os seus compromissos (OLIVEIRA; SUCHARA, 2014a). Além disso, as mulheres comumente adotam hábitos de saúde em maior frequência que os homens e, portanto, utilizam mais medicamentos, estando mais susceptíveis a intoxicação por esses agentes (MENDONÇA et al., 2005; GANDOLFI; ANDRADE, 2006; SOUTO et al., 2013).

A alta prevalência de eventos toxicológicos relacionados aos medicamentos por mulheres também é relatada por outros autores: no Estado de São Paulo (GANDOLFI; ANDRADE, 2006; ARRAIS et al., 2005), em uma cidade do Sul do Brasil (MARGONATO et al., 2009); em Uberlândia, Minas Gerais (SANTOS et al., 2015) e na Bahia (TELES et al., 2013).

As intoxicações registradas em Primavera do Leste - MT ocorreram com maior frequência nas faixas etárias entre 20 a $39 \operatorname{anos}(50 \% ; n=26)$ e 15 a $19(17,3 \% ; n=9)$ (Figura 1). Semelhante ao registrado para o presente estudo, Teles et al. (2013) encontraram que a faixa etária mais acometida em todos os anos de sua pesquisa foi a de 20 a 34 anos, seguida de 1 a 4 , de 15 a 19 e de 35 a 49 anos, respectivamente.

Figura 1 - Número de casos de intoxicação medicamentosa por faixa etária, no município de Primavera do Leste - MT, 2007 a 2014.

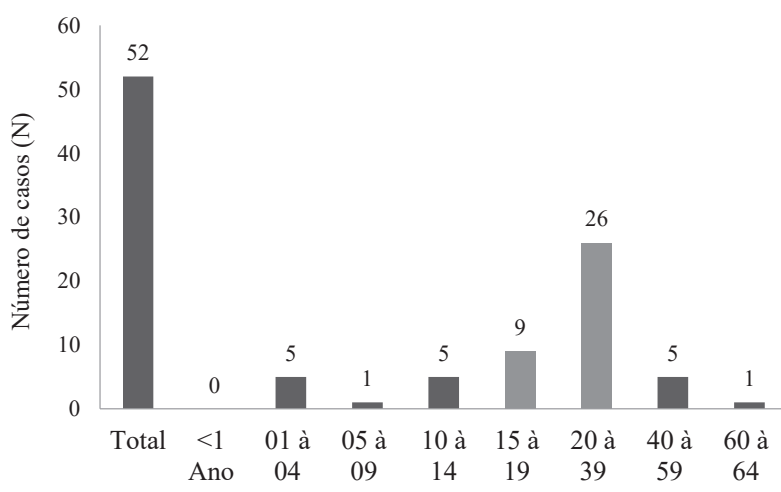

Faixa etária das vítimas de intoxicação medicamentosa

Fonte: Dados da pesquisa

Oliveira et al. (2017) avaliaram as magnitudes dos óbitos relacionados ao uso de medicamentos, observou-se maior tendência de mortalidade por intoxicação medicamentosa para os adolescentes (15 a 19 anos) e para os adultos jovens (20 a 29 anos), seguidos por adultos (30 a 39 anos e 40 a 49 anos) e idosos (idade superior a 70 anos).

Santos et al. (2015) também registraram a maioria dos casos nas faixas etárias de jovens adultos entre 18 a 49 anos. Conforme proposto por Oliveira e Suchara (2014a), as faixas etárias mais frequentemente envolvidas são de adultos em idade produtiva, o que também pode estar relacionado à prática comum de automedicação dos indivíduos dessa faixa em função da necessidade de resolver mais rapidamente os problemas de saúde para manter a qualidade do trabalho. De acordo com Santos et al. (2015), os elevados percentuais de intoxicações intencionais nos indivíduos de 20 a 39 anos podem ser relacionados aos obstáculos encontrados, intrínsecos a esta idade, como dificuldade de inserção no mercado de trabalho e problemas pessoais e familiares, podendo estar ligados aos 
transtornos depressivos não tratados.

Os dados de intoxicações por diversos agentes tóxicos registradas para o município, entre esses os medicamentos, destacaram que a maioria das vítimas envolvidas é das raças/ cor parda e branca e possuem o Ensino Fundamental como nível de escolaridade. A ocorrência em maior proporção de indivíduos das raças pardas e branca, possivelmente, está relacionada predomínio de indivíduos dessas raças no município de Primavera do Leste- MT, conforme dados registrados pelo Instituto Brasileiro de Geografia e Estatística.

Níveis de escolaridade baixos também foram observados em outros estudos de casos de intoxicação (SANTANA et al., 2016; MELO; SILVA, 2013). A desinformação e o desconhecimento, por parte da população, sobre o uso correto de medicamentos, pode acarretar risco de intoxicações relacionadas a esses produtos (OLIVEIRA; SUCHARA, 2014b)

As principais circunstâncias observadas foram: tentativa de suicídio $(73,1 \% ; n=38)$, acidental $(15,4 \% ; n=8)$ e as demais por uso habitual, automedicação, abuso e violência/homicídio (Figura 2).

Figura 2 - Número de casos por circunstâncias das intoxicações por medicamentos registradas para o município de Primavera do Leste - MT, entre 2007 a 2014.

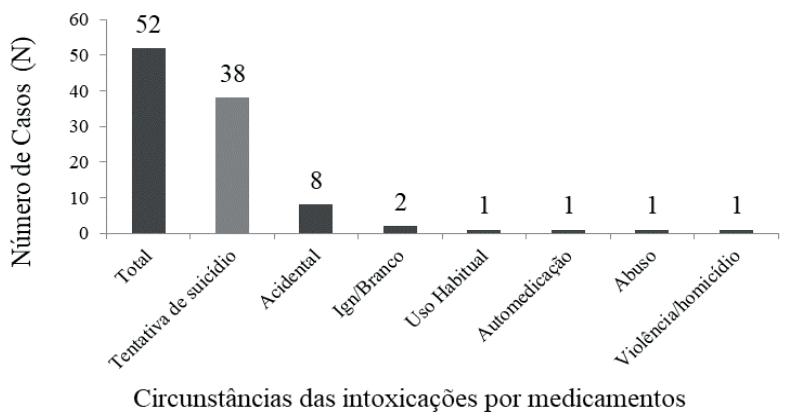

Fonte: Dados da pesquisa

Resultados semelhantes aos encontrados para o presente estudo, quanto aos casos de intoxicações intencionais, por tentativa de suicídio, também foram registrados no estudo de Bertasso-Borges et al. (2010), Margonato et al., (2009), Teles et al. (2013) e Oliveira e Suchara (2014a,b), em que as tentativas de suicídio com uso de medicamentos também representaram os casos mais comuns entre as intoxicações.

$\mathrm{O}$ aumento dos índices de tentativas de suicídio tem sido observado em várias localidades de todo mundo, principalmente, envolvendo adolescentes e adultos jovens (REGADAS et al., 2000; SPILLER et al., 2010). Vários são os fatores atuais relacionados ao crescimento do índice dessa circunstância, entre eles estão os conflitos em relacionamentos afetivo, na escola, doença mental, estruturas familiares perturbadas (SCHMIDT et al., 2002) fácil acesso aos medicamentos e por uma provável instabilidade emocional, o que intencionalmente leva aumentam o risco da adoção de medidas com a intenção de por fim à vida (OLIVEIRA; SUCHARA, 2014a).
A relevância das tentativas de suicídio reforça a necessidade de intensificar ações de atenção em saúde mental, especializando ações multiprofissionais de promoção à saúde destinada à prevenção deste agravo (OLIVEIRA; SUCHARA, 2012; REIS et al., 2013).

Conforme demonstrados através dos estudos de Bernardes et al. (2010), Mota et al. (2012), Bochner e Souza (2008) e Bochner (2006), os adultos jovens e indivíduos do sexo feminino são as vítimas mais vulneráveis aos casos de tentativa de suicídio com abuso de medicamentos. De acordo com Kaplan et al. (1997), o sexo feminino é quatro vezes mais propenso a tentar o suicídio do que o sexo masculino.

No estudo de caracterização de tentativas de suicídio realizado por Vieira et al. (2015), também foi registrado que a maioria das vítimas pertencia ao gênero feminino. No mesmo estudo, os autores encontraram que os adultos (46,7\%) e os adolescentes $(72,7 \%)$ também estiveram mais frequentemente envolvidos nas tentativas de suicídio predominantemente com uso de medicamento, o que pode ser decorrente de essas faixas etárias terem mais acesso aos medicamentos.

Zambolim et al. (2008) abordam que, de acordo com a Organização Mundial de Saúde (OMS), os casos de intoxicação podem ser um acidente ou uma tentativa deliberada de assassinato ou de suicídio. Portanto, tanto as intoxicações acidentais quanto as intencionais constituem importantes causas de problemas de saúde.

Os casos acidentais vitimaram indivíduos em maior proporção do sexo masculino, nas faixas etárias entre 20 a 39 anos, 0 a 04 e 10 a 14 anos. Teles et al. (2013) também registraram os acidentes como circunstâncias mais frequentes, assim como na pesquisa de Bortoletto e Bochner (1999), que avaliou o impacto dos medicamentos na intoxicação exógena, as circunstâncias acidentais foram as que registraram maior número de casos $(60 \%)$, sendo a faixa etária mais frequente a de crianças menores de cinco anos (33\%), seguida da faixa etária de 20 a 29 anos (19\%), semelhante ao registrado para o presente estudo.

Dados do SINITOX referentes ao período de 2003 a 2005, apontam as crianças menores de cinco anos como as maiores vítimas dos casos de intoxicação por medicamentos (31,8\%) (FIOCRUZ, 2000). O que é devido às crianças, nesta faixa etária, mais comumente levarem substâncias ou objetos coloridos à boca (HAHN, 2013), além disso, estas crianças começam a andar e desenvolvem a necessidade de provar em descobrir o novo. Dessa forma, os comportamentos relacionados ao crescimento e desenvolvimento infantil podem favorecer a ocorrência desses acidentes (KOLIOU et al., 2010). Outros fatores como diferenças na farmacocinética e farmacodinâmica dos medicamentos também tornam as crianças mais susceptíveis às intoxicações por medicamentos (BERTASSO-BORGES, 2010).

Muitos dos casos de intoxicação envolvendo crianças se deve, principalmente, a administração errônea por um adulto (MARCHI et al., 1998). No entanto, conforme proposto 
por Lessa e Bochner (2008), a automedicação, assim como a medicação leiga feita pelos pais sem orientação médica, constituem circunstâncias intencionais, e não deveriam ser consideradas como acidentais.

Em geral, as intoxicações exógenas em crianças menores de cinco anos são acidentais, passíveis de prevenção, decorrentes de situações facilitadoras das características peculiares das fases de desenvolvimento da criança e do pouco incentivo às medidas preventivas (SHANNON, 2000). Nos estudos desenvolvidos por Chien et al. (2003), Lam (2003) e Baracat et al. (2000), as substâncias farmacêuticas têm sido a categoria mais frequente de intoxicação não-intencional na infância.

Segundo Schvartsman e Schvartsman (1999), quando se considera a faixa etária pediátrica, as intoxicações agudas assumem um papel de destaque. Assim sendo, a adoção de programas educacionais e preventivos de intoxicações acidentais em escolas, creches, e nas comunidades, associados à utilização de embalagens mais seguras à abertura pela criança, aos cuidados com o armazenamento de medicamentos e produtos químicos de uso doméstico, pode contribuir para a diminuição dos casos de intoxicação acidental por medicamentos em crianças (BORTOLETTO; BOCHNER, 1999).

A exposição aguda única foi a mais frequente, representando $82,7 \%$ dos casos $(n=43)$. Houve cinco casos de exposição aguda repetida e nenhum de exposição crônica (Quadro 1). Conforme os dados de intoxicação por diversos agentes tóxicos para o município, as exposições agudas registradas se deram principalmente por tentativa de suicídio, acidentes e uso habitual e os casos de exposição aguda repetida ocorreram em maior frequência de forma acidental, seguida de tentativa de suicídio.

Muitos fármacos não ocasionam lesões patológicas características, sendo os sintomas de intoxicação, muitas vezes, inespecíficos (KLAASSEN; WATKINS, 2001), dessa forma, não havendo um diagnóstico confirmado, os pacientes comumente recebem apenas tratamento sintomático (AMARAL; BARCIA, 2003). Além disso, há tendência de se registrar apenas os casos agudos, o que de acordo com Oliveira et al. (2017) podem subestimar as estimativas por não incluírem os casos de uso continuado de medicamentos.

A maioria dos casos evoluiu para cura sem sequelas $(84,6 \% ; n=44)$ e em um caso registrou-se óbito $(1,9 \%)$, nos demais foram ignorados os registros de evolução (Quadro 1). No estudo realizado por Santos e Boing (2018), em que avaliaram as intoxicações e reações adversas aos medicamentos no Brasil durante o período de 2000 a 2014, registraram aproximadamente $0,1 \%$ dos óbitos e a maioria desses estiveram relacionados às intoxicações medicamentosas.

No estudo desenvolvido por Vieira et al. (2015), que avaliou os casos de intoxicação por tentativa de suicídio em um município de Mato Grosso, também foi maior a frequência de exposição aguda única $(75,6 \%)$, seguida pela aguda repetida. Em 76,2\% dos casos as vítimas evoluíram para cura sem sequelas e dos quatro óbitos, três indivíduos eram do sexo masculino, semelhante ao registrado para o município de Primavera do Leste - MT.

No trabalho de Bortoletto e Bochner (1999) sobre os impactos dos medicamentos nas intoxicações humanas no Brasil, a segunda causa mais frequente de óbitos ocorreu por intoxicação medicamentosa e em $56 \%$ foram atribuídos à circunstância "suicídio". Santos e Boing (2018) também registraram que a principal causa de óbito decorrente de autointoxicação por exposição intencional ocorreu pela utilização de medicamentos, além de outras drogas e substâncias biológicas não especificadas.

Os dados disponibilizados pelo SINAN não informam as principais classes de medicamentos envolvidas nos acidentes registrados para o presente estudo, pois nas fichas de notificação não há um campo específico para o preenchimento dessa informação. Dessa forma, o sistema não conta com o registro desses dados, ficando esses anotados apenas na ficha de atendimento médico de cada paciente e arquivadas nas unidades de atendimento de saúde.

Porém, conforme dados do SINITOX, apresentados por Bartoletto e Bochner (1999), os benzodiazepínicos, antigripais, antidepressivos, anti-inflamatórios são as classes de medicamentos que mais causam intoxicações no país. Conforme abordado por Silva et al. (2011), as intoxicações das leves às graves, as mais comuns são provocadas por medicamentos, principalmente os antipsicóticos.

Conforme o estudo de Oliveira et al. (2017), o uso de narcóticos e psicodislépticos foram as causas principais de óbitos por intoxicação medicamentosa acidental para o gênero masculino, já para o feminino foram em função de uso de anticonvulsivantes, sedativos, hipnóticos, antiparkinsonianos e psicotrópicos, sendo esses classificados como intencionais. Conforme evidenciado por Townsend et al. (2001), os psicoativos, principalmente os tranquilizantes, antidepressivos e anticonvulsivos, possuem alta participação entre os medicamentos utilizados nas tentativas de suicídio. No estudo do perfil das tentativas de suicídio por overdose de medicamentos desenvolvido por Bernardes et al. (2010), esses medicamentos corresponderam a $57,5 \%$ dos casos estudados e entre os medicamentos de venda livre se destacou a dipirona como a substância mais frequente.

Em um estudo de intoxicações exógenas em crianças atendidas em uma unidade de emergência pediátrica, os anticonvulsivantes e os broncodilatadores tiveram o maior número de casos (LOURENÇO et al., 2008). No trabalho de Matos et al. (2002), em que se avaliou intoxicações medicamentosas em crianças com menos de cinco anos, foram identificados cinco grupos de medicamentos responsáveis pelo maior número de casos: os descongestionantes nasais, os analgésicos, os broncodilatadores, os anticonvulsivantes e os 
contraceptivos orais.

Pesquisa realizada por Lessa e Bochner (2008) registrou que os antiepilépticos, sedativos hipnóticos e antiparkinsonianos foram responsáveis por $15,2 \%$ das internações relacionadas às intoxicações e aos efeitos adversos de medicamentos em menores de um mês e $21,1 \%$ em crianças de um a onze meses. Os antibióticos sistêmicos responderam para essas mesmas faixas etárias por $12,6 \%$ e $18,5 \%$ das internações, respectivamente. Os analgésicos, antitérmicos e antirreumáticos não opiáceos tiveram participação menor, com $7,3 \%$ das internações.

O amplo uso das classes de medicamentos citadas, o desconhecimento dos malefícios do uso irracional e sem orientação médica, constituem as principais causas dos medicamentos serem os agentes tóxicos responsáveis pelas intoxicações humanas registradas no país (LESSA; BOCHNER, 2008).

Assim, o conhecimento dos registros de intoxicação por medicamento, bem como do perfil desses casos no município em estudo e demais localidades próximas é fundamental para auxiliar na elaboração de políticas preventivas e para adoção das práticas de uso racional de medicamentos, a fim de proporcionar maior qualidade de vida para a população.

\section{Conclusão}

Os medicamentos representam um dos principais agentes de intoxicações humanas registradas no Brasil e também no município estudado. Ao longo dos anos avaliados foram notificados 52 casos de intoxicações por medicamentos em Primavera do Leste- MT, as vítimas mais frequentemente envolvidas foram do gênero feminino, nas faixas etárias entre 20 a 39 e 15 a 19 anos, raça parda e branca e apenas com Ensino Fundamental. A exposição aguda única foi a mais frequente e os casos ocorreram, principalmente, por tentativa de suicídio, acidental e uso habitual. A maioria dos casos evoluiu para cura sem sequelas.

Assim, as intoxicações medicamentosas constituem um importante problema de saúde pública e as ações preventivas e de educação em saúde devem ser direcionadas aos grupos populacionais específicos, ajudando a reduzir o impacto das ocorrências de intoxicações medicamentosas nas unidades de emergência e no Sistema de Saúde como um todo. Deve-se, ainda, buscar garantir o uso correto e racional de medicamentos e, desta forma, contribuir para a redução dos casos em adultos jovens, especialmente, em indivíduos do gênero feminino que, de acordo com o presente estudo, estão mais susceptíveis a sofrerem intoxicações.

\section{Referências}

AMARAL, D.A.; BARCIA, S.A.D. Intoxicações por medicamentos. In: OGA, S. organizador. Fundamentos de toxicologia. São Paulo: Varella, 2003. p.367-379.

ARRAIS, P. S. D. O uso irracional de medicamentos e a farmacovigilância no Brasil. Cad. Saúde Pública, v.18, n.5, p.1478-1479, 2002. doi: 10.1590/S0102-311X2002000500042

ARRAIS, P.S.D. et al. Prevalence and determinants of medicines consumption in Fortaleza, Ceará, Brazil. Cad Saúde Pública. v.21, p.1737-1746, 2005. doi: 10.1590/S0102-311X2005000600021.

BARACAT, E.C.E. et al. Acidentes com crianças e sua evolução na região de Campinas, SP. J. Pediatr. v.76, n.5. p.368-374, 2000. doi: 10.1590/S0102-311X2002000500042

BERNARDES, S.S.; TURINI, C.A.; MATSUO, T. Perfil das tentativas de suicídio por sobredose intencional de medicamentos atendidas por um Centro de Controle de Intoxicações do Paraná, Brasil. Cad Saúde Pública. v.26, n.7, p.1366-1372, 2010. doi: 10.1590/S0102-311X2010000700015.

BERTASSO-BORGES, M.S. et al. Eventos toxicológicos relacionados a medicamentos registrados no CEATOX de São José do Rio Preto, no ano de 2008. Arq. Cienc. Saúde, v.17, n.1, p.35-41, 2010.

BOCHNER, R. Perfil das intoxicações em adolescentes no Brasil no período de 1999 a 2001. Cad Saúde Pública, v.22, n.3, p.58795, 2006. doi: 10.1590/S0102-311X2006000300014.

BOCHNER, R.; SOUZA, V.M.F.A. Panorama das intoxicações e envenenamentos registrados no Brasil pelo Sistema Nacional de Informações Tóxico-Farmacológicas (SINITOX). Rev. Racine, v.18, n.106, p.44-58, 2008.

BORTOLETTO, M.; BOCHNER, R. Impacto dos medicamentos nas intoxicações humanas no Brasil. Cad. Saúde Pública, v.15, n.4, p.859-69, 1999. doi: 10.1590/S0102-311X1999000400020.

BRASIL. Casos registrados de intoxicação humana, de intoxicação animal e solicitações de informações por agente tóxico, Brasil, 2011. Sistema de Informação Tóxico-Farmacológicas (SINITOX), 2011b.

BRASIL. Portaria $\mathrm{n}^{\circ} 104$ de 26 de janeiro de 2011. DOU N ${ }^{\circ} 18$, seção I, p. 37 e 38, quarta-feira, 26 de janeiro de 2011. Brasília: MS, 2011a.

BRASIL. Vigilância sanitária, alimentos, medicamentos, produtos e serviços de interesse a saúde - guia didático. Brasília: Agência Nacional de Vigilância Sanitária e Instituto de Defesa do Consumidor, 2007.

CHIEN, C. et al. Unintentional ingestion of over the counter medications in children less than 5 years old. J. Pediatr. Child Health, v.39, n.4, p.264-269, 2003. doi: 10.1046/j.14401754.2003.00148.x

COELI, C.M.; CAMARGO JR, K.R. Avaliação de diferentes estratégias de blocagem no relacionamento probabilístico de registros. Rev. Bras. Epidem., v.5, n.2, p.185-196, 2002. doi: 10.1590/S1415-790X2002000200006.

FIOCRUZ. Casos registrados de intoxicação humana por agente tóxico e faixa etária. Região Centro-oeste. 2010 a. Disponível em: http:// www.fiocruz.br/sinitox_novo/media/co5.pdf. Acesso em: 20 abr. 2019.

FIOCRUZ . Casos registrados de intoxicação humana por agente tóxico e sexo. Região Centro-oeste. 2010b. Disponível em: http:// www. fiocruz.br/sinitox_novo/media/co6.pdf. Acesso em: $20 \mathrm{abr}$. 2019.

FIOCRUZ. Casos registrados de intoxicação humana por agente tóxico e circunstância. Região centro-oeste. 2010c. Disponível em: http:// www.fiocruz.br/sinitox_novo/media/co4.pdf. Acesso em: 20 abr. 2019.

FIOCRUZ. Estatística Anual de casos de intoxicação e envenenamento. Brasil, 2003, 2004, 2005. Centro de Informação Científica e Tecnológica. Sistema Nacional de Informações Tóxico-Farmacológicas. Disponível em: http://www.fiocruz.br/ 
sinitox. Acesso em: 10 abr. 2019.

GANDOLFI, E.; ANDRADE, M.G. Drug-related toxic events in the state of São Paulo, Brazil. R. Saúd. Pública, v.40, p.1056-64, 2006. doi: 10.1590/s0034-89102006000700014

GANDOLFI, E.; ANDRADE, M.G.G. Eventos toxicológicos relacionados a medicamentos no Estado de São Paulo. Rev. Saúd. Pública, v.40, n.6, p.1056-1064, 2006. doi: 10.1590/S003489102006000700014

HAHN, R.C.; LABEGALINI, M.P.; OLIVEIRA, M.L. Features of acute poisoning in children: a study in center toxicological. BJSCR, v.4, n. 1, p. 18-22, 2013.

KAPLAN, H.I; SADOCK, B.J.; GREBB, J.A. Compêndio de psiquiatria: ciências do comportamento e psiquiatria clínica Porto Alegre: Artmed, 1997.

KLAASSEN, C.D.; WATKINS, III J.B. Toxicologia, A ciência básica dos tóxicos de Cassarett e Doull. Alfragide: McGraw-Hill de Portugal, 2001.

KOLIOU, M. et al. The epidemiology of childhood poisonings in Cyprus. Eur. J. Pediatr., n.169, p.833-8, 2010. doi: 10.1007/ s00431-009-1124-8

LAM, L.T. Childhood and adolescence poisoning in NSW, Australia: an analysis of age, sex, geographic, and poison types. Inj Prev. v.9, n.4, p.338-42, 2003. doi: 10.1136/ip.9.4.338

LESSA, M.A.; BOCHNER, R. Análise das internações hospitalares de crianças menores de um ano relacionadas a intoxicações e efeitos adversos de medicamentos no Brasil. Rev. Bras. Epidemiol., v.11, n.4, p.660-674, 2008. doi: 10.1590/ S1415-790X2008000400013

LOURENÇO, J.; FURTADO, B.M.A.; BONFIM, C. Intoxicações exógenas em crianças atendidas em uma unidade de emergência pediátrica. Acta Paul Enferm., v.21, n.2, p.282-286, 2008. doi: 10.1590/S0103-21002008000200008

MARCHI, A.G. et al. Childhood poisoning: a population study in Trieste, Italy, 1975-1994. J. Clin. Epidemiol., n.51, p.687-695, 1998. doi: 10.1016/S0895-4356(98)00045-6

MARGONATO, F.B.; THOMSON, Z.; PAOLIELLO M.M.B. Acute intentional and accidental poisoning with medications in a southern Brazilian city. Cad. Saúde Pública, v. 25, n. 4, p. 849-56 2009. doi: 10.1590/S0102-311X2009000400016

MATOS, C.G.; NASCIMENTO A.C. Impactos dos medicamentos como agentes de intoxicações humanas. Rev. Racine. n.106, v.5, p.59-66, 2008.

MATOS, G.C.; ROZENFELD, S.; BORTOLETTO, M.E. Intoxicações medicamentosas em crianças menores de cinco anos. Rev. Bras. Saúde Mat. Infantil, v.2, n.2, p.167-176, 2002. doi: 10.1590/S1519-38292002000200009.

MENDONÇA, R.T.; MARINHO, J.L. Discussão sobre intoxicações por medicamentos e agrotóxicos no Brasil de 1999 a 2002. Rev. Eletr. Farm., v.2, n.2, p.45-63, 2005. doi: 10.5216/ ref.v2i2.1950

MERT, E.; BILGIN, N.G. Demographical, a etiological and clinical characteristics of poisonings in Mersin, Turkey. Hum. Exp. Toxicol., n.25, p.217-223, 2006. doi: 10.1191/0960327106ht612oa

MOREIRA, C.S. et al. Análise retrospectiva das intoxicações admitidas no hospital universitário da UFJF no período 20002004. Ciênc. Saúd. Col., v.15, n.3, p. 879-888, 2010. doi: 10.1590/ S1413-81232010000300031.

MOTA, D.M. et al. Perfil da mortalidade por intoxicação com medicamentos no Brasil, 1996-2005: retrato de uma década Ciênc. Saúd. Col., v.17, n.1, p.61-70, 2012. doi: 10.1590/S1413-

\section{9}

OGA, S.; CAMARGO, M.M.A; BATISTUZZO, J.A.O Fundamentos de toxicologia. São Paulo: Atheneu, 2008.

OLIVEIRA, C.S.; FERREIRA, A.P. Perfil epidemiológico das ações de vigilância em saúde das populações expostas aos agrotóxicos. Interfaches, v.7, n.1, p.1-16, 2012.

OLIVEIRA, D.H.; SUCHARA, E.A. Intoxicações medicamentosas em hospital público de Barra do Garças -MT, no período de 2006 a 2009. Rev. Ciênc. Méd. Biológ., v.13, n.1, p.55-59, 2014a.

OLIVEIRA, F.F.S.; SUCHARA, E.A. Perfil epidemiológico das intoxicações exógenas em crianças e adolescentes em município do Mato Grosso. Rev. Paul. Pediatr., v.32, n.4, p.299-305, 2014 b. doi: 10.1590/S0103-05822014000400004

OLIVEIRA, J.F.M. et al. Tendência da mortalidade por intoxicação medicamentosa entre gêneros e faixas etárias no Estado de São Paulo, Brasil, 1996-2012. Ciênc. Saúde Col., v.22, n.10, p.33813391, 2017. doi:10.1590/1413-812320172210.12782017

PRESGRAVE, R.F.; CAMACHO, L.A.; VILLAS BOAS, M.H. Analysis of data from Poison Control Centers in Rio de Janeiro, Brazil, for use in public health activities. Cad. Saúd. Públ., n.25, p.401-408, 2009. doi: 10.1590/S0102-311X2009000200019

REGADAS, R.P. et al. Suicide attempt by self-poisoning: a retrospective study of 446 cases. Pesq. Med., n.3, p.3-50, 2000.

REIS, L.M. et al. Saúde do homem: internações hospitalares por intoxicação registradas em um Centro de Assistência Toxicológica. Esc. Anna Nery, v.17, n.3, p.505-511, 2013. doi: 10.1590/S1414-81452013000300014.

ROZENFELD, S. Farmacovigilância: elementos para discussão e perspectivas. Cad. Saúde Pública, n.14, p 63-237, 1998. doi: 10.1590/S0102-311X1998000200002.

SANTOS, R.R.; ALMEIDA NETO, O.P.; CUNHA, C.M. Perfil de vítimas de intoxicações exógenas agudas e assistência de enfermagem. Rev. Enferm. Atenção Saúde, v.4, n.2, p.45-55, 2015.

SANTOS, G.A.S.; BOING, A.C. Hospitalizations and deaths from drug poisoning and adverse reactions in Brazil: an analysis from 2000 to 2014. Cad. Saude Publica, v.34, n.6, p.00100917-e00100917,2018. doi: 10.1590/0102-311X00100917

SCHMIDT, P. et al. Suicide in children, adolescents and young adults. Forensic Sci Int., n.127, p. 161-7, 2002. doi: https://doi. org/10.1016/S0379-0738(02)00095-6

SCHVARTSMAN, C.; SCHVARTSMAN, S. Intoxicações exógenas agudas. J. Pediatr. Soc. Bras. Pediatr., v.75, Supl.2, 1999.

SHANNON, M. Ingestion of toxic substances by children. N. Engl. J. Med., v.342, p.186-191, 2000. doi: 10.1056/ NEJM200001203420307

SILVA, C.C.S.; SOUZA, K.S.; MARQUES, M.F.L. Intoxicações Exógenas: Perfil dos Casos que Necessitaram de Assistência Intensiva em 2007. Rev. Bras. Ciênc. Saúde, v.15, n.1, p.65-68, 2011. doi: 10.4034/RBCS.2011.15.01.09.

SILVA, IG. SIH-SUS como fonte para o estudo de intoxicações causadas por medicamentos no estado do Rio de Janeiro de 1999 a 2007. Rio de Janeiro: Universidade do Estado do Rio de Janeiro, 2009.

SINITOX - Sistema Nacional de Informações TóxicoFarmacológica. Estatística anual dos casos de intoxicação e envenenamento. Brasil. 1997. Rio de Janeiro: FIOCRUZ, 1998. 
SOUTO, C.E. et al. Intoxicações medicamentosas em Araucária PR. Rev. Uniandrade, v.13, n.3, p.210-220, 2013.

SPILLER, H.A.; APPANA, S.; BROCK, G.N. Epidemiological trends of suicide and attempted suicide by poisoning in the US: 2000-2008. Leg Med. Tokyo, n.12, p.177-83, 2010. doi: 10.1016/j. legalmed.2010.04.005.

TELES, A.S. et al. Papel dos medicamentos nas intoxicações causadas por agentes químicos em município da Bahia, no período de 2007 a 2010. Rev. Ciênc. Farm. Básica Apl., v.34, n.2, p.281-288, 2013.

TOWNSEND, E. et al. Substances used in deliberate selfpoisoning 1985-1997: trends and associations with age, gender, repetition and suicide intent. Soc. Psychiatr. Psychiatr. Epidemiol., v.36, n. 5, p. 228-34, 2001. doi: 10.1007/s001270170053.

VIEIRA, L.P.; SANTANA, V.T. P.; SUCHARA, E.A. Caracterização de tentativas de suicídios por substâncias exógenas. Cad. Saúde Colet., v.23, n.2, p.118-123, 2015. doi: 10.1590/1414-462X201500010074.

WONG, A. Os usos inadequados e os efeitos adversos de medicamentos na prática clínica. J. Pediat., v.79, n.5, p.379-380, 2003.

ZAMBOLIM, C.M. et al. Exogenous intoxications profile in a university hospital. Rev. Med. Minas Gerais, v.18, n.1, p.5-10, 2008. 